\title{
Role of Gallbladder Mucus Hypersecretion in the Evolution of Cholesterol Gallstones
}

\author{
STUDIES IN THE PRAIRIE DOG
}

\author{
Sum P. Lee, J. Thomas LaMont, and Martin C. Carey, Department of Medicine, \\ Harvard Medical School, Division of Gastroenterology, Peter Bent Brigham \\ Hospital, Division of Brigham and Women's Hospital, Inc., \\ Boston, Massachusetts 02115
}

\begin{abstract}
A B S T R A C T Because mucin glycoproteins may be important in the pathophysiology of gallstones, we studied the relationship among biliary lipids, gallbladder mucin secretion, and gallstone formation in cholesterol-fed prairie dogs. Organ culture studies of gallbladder explants revealed that the incorporation of $\left[{ }^{3} \mathrm{H}\right]$ glucosamine into tissue and secretory gallbladder glycoproteins was significantly increased at $3,5,8$, and $14 \mathrm{~d}$ of feeding. Peak secretion of labeled mucin occurred at $5 \mathrm{~d}$, when total tissue and secreted glycoprotein production was fivefold greater than control. Gel filtration of the secreted glycoprotein on Sepharose 4B indicated that the majority of radioactivity was present in a macromolecule of $>1$ million molecular weight. The increased secretion of gallbladder mucin was organ specific, in that $\left[{ }^{3} \mathrm{H}\right]-$ glucosamine incorporation into glycoproteins of stomach and colon was unaffected by cholesterol feeding. Similarly, the incorporation of $\left[{ }^{3} \mathrm{H}\right]$ mannose into gallbladder membrane glycoproteins was not altered by cholesterol feeding. The rate of glycoprotein synthesis and secretion returned to normal upon withdrawal of the cholesterol diet, and ligation of the cystic duct before cholesterol feeding prevented gallbladder mucin hypersecretion. Both results indicate that the stimulus to mucin secretion was a constituent of bile. Gallbladder bile after $5 \mathrm{~d}$ contained cholesterol in micelles, liquid crystals, and crystals, whereas
\end{abstract}

Part of this study was presented at the Annual Meeting of the American Gastroenterological Association, New Orleans, and published as an abstract in 1979. Gastroenterology. 76: 1183.

Dr. Lee's current address is Division of Gastroenterology, Auckland Hospital, Grafton, New Zealand. Address correspondence to Dr. Thomas LaMont, University Hospital, Boston, Mass.

Received for publication 7 August 1980 and in revised form 26 January 1981. hepatic bile remained a single micellar phase throughout cholesterol feeding. For this reason the cholesterol-saturation indices of gallbladder bile were compared in both homogenized and centrifuged samples. The micellar phase of gallbladder bile was appreciably less saturated than homogenized bile at 5 and $8 \mathrm{~d}$, which reflects the continuous nucleation of cholesterol in the gallbladder. Purified human gallbladder mucin gels were shown to induce nucleation of lecithin-cholesterol liquid crystals from supersaturated hepatic bile. These in turn gave rise to cholesterol monohydrate crystals within $18 \mathrm{~h}$. Control supersaturated hepatic bile could not be nucleated by the addition of other proteins, and was stable for days upon standing. These results suggest that the increase in cholesterol content of bile in cholesterolfed prairie dogs stimulates gallbladder mucus hypersecretion, and that gallbladder mucus gel is a nucleating agent for biliary cholesterol.

\section{INTRODUCTION}

The formation of cholesterol gallstones requires at least three sequential physicochemical steps: hepatic secretion of cholesterol-supersaturated bile; nucleation and precipitation of excess cholesterol from biliary micelles; and retention, agglomeration, and eventual growth of cholesterol monohydrate crystals into macroscopic stones (1). Supersaturation of hepatic and gallbladder bile has been well documented in gallstone patients $(2,3)$, as well as in obese subjects (4), patients with interrupted enterohepatic cycling of bile salts (5), and normal individuals, especially during fasting $(2,3,6)$. Supersaturated gallbladder bile, however, does not necessarily result in the formation of cholesterol crystals or gallstones $(2,3)$. Previous studies (7-10) emphasized the importance of choles- 
terol monohydrate crystals in bile as a means of distinguishing lithogenic from nonlithogenic biles. Recently, Holan et al. (11) demonstrated that supersaturated bile from gallstone patients differs from supersaturated bile from patients without stones in its ability to nucleate micellar cholesterol. In addition, Sedaghat and Grundy (12) have confirmed both of these observations by observing that cholesterol monohydrate crystals in the bile or the ability of bile to grow cholesterol crystals was invariably associated with cholesterol gallstones. An obvious corollary of these studies is that either a nucleating factor is present in lithogenic bile or substances which inhibit nucleation are present in supersaturated bile from normal individuals. If a "nucleation factor" is present, it most likely arises from the gallbladder itself since cholesterol gallstones are common in the gallbladder, rare in the bile ducts, and occur infrequently after cholecystectomy even though hepatic bile may remain supersaturated $(13,14)$.

That gallbladder mucus may be of crucial importance in the pathogenesis of gallstones, particularly as a nucleating agent, was first emphasized by Womack et al. (15) and Hultén (16). Womack later noted (17) that gallbladder mucus hypersecretion preceded gallstone development in hamsters fed a lithogenic diet. After also observing that cholesterol crystals first formed in aggregates of mucus rather than in the liquid phase of gallbladder bile, he hypothesized that a prosthesis of mucus was essential for the development of cholesterol gallstones. That mucus may also be important in the pathophysiology of other types of gallstones has also been suggested. For example, Freston et al. (18) found increased biliary hexosamine owing to elevated glycoprotein concentrations in gallbladders of rabbits fed $5 \alpha$-cholestanol before the appearance of glycoallodeoxycholate stones (19). Similarly, Englert et al. (20) noted increased mucus secretion during diet-induced pigment gallstones in the dog. Further studies by Bouchier et al. (21) and Lee et al. (22) demonstrated that gallbladder bile from patients with gallstones contained more glycoproteins and was more viscous than normal owing to its greater concentration of mucus. Although the role of cholesterol-supersaturated bile in the pathophysiology of cholesterol gallstones is well established, the interrelationships between mucus synthesis and secretion and the lipid composition of bile has not been studied in detail.

To study these relationships, we chose the cholesterol-fed prairie dog, an excellent model of cholesterol gallstone formation (23-26). We quantified the relationship between gallbladder mucus synthesis and secretion and the formation of cholesterol gallstones using an organ culture technique. By measuring the incorporation of $\left[{ }^{3} \mathrm{H}\right]$ glucosamine into gallbladder glycoproteins during the development and nucleation of supersaturated bile, we found a marked increase in mucus synthesis and secretion with increasing relative cholesterol content of bile. Cholesterol crystals and their growth into gallstones took place predominantly in a mucus gel which was adherent to the gallbladder wall.

\section{METHODS}

Cholesterol (USP grade, Sigma Chemical Co., St. Louis, Mo.) was twice recrystallized from hot $95 \%$ ethanol and stored under nitrogen. This material, migrated as a single spot by thin-layer chromatography, had a melting point of 149$150^{\circ} \mathrm{C}$ and was at least $99 \%$ pure by gas-liquid chromatography. Sodium taurocholate (Calbiochem-Behring Corp., San Diego, Calif.) was recrystallized from ether and aqueous ethanol and migrated as a single peak on thin-layer chromatography and a single peak by high performance liquid chromatography. Lecithin (egg yolk, grade A, Lipid Products, Surrey, England) was $99 \%$ pure by thin-layer chromatography in several solvent systems $(2,27)$ and was used without further purification. Glucosamine and $5 \alpha$-cholestanol (chromatography grade standards) were obtained from Supelco, Inc., Bellefonte, $\mathrm{Pa}$. and $\mathrm{D}-\left[6{ }^{3} \mathrm{H}\right]$ glucosamine hydrochloride (specific activity, $10-30 \mathrm{Ci} / \mathrm{mmol}$ and $\mathrm{D}-\left[2-{ }^{3} \mathrm{H}\right]$ mannose (specific activity, $10 \mathrm{Ci} / \mathrm{mmol}$ ) was obtained from New England Nuclear, Boston, Mass. Sepharose 4B and protein standards for chromatography were obtained from Pharmacia Fine Chemicals, Div. Pharmacia Inc., Piscataway, N. J. Organ culture medium (Trowell's T-8) was obtained from Grand Island Biological Company, Grand Island, N. Y., and organ culture grids and dishes were from Falcon Labware, Div. Becton, Dickinson \& Co., Oxnard, Calif. Scintillation solvent (Redisolv-HP) was a product of Beckman Instruments, Inc., Palo Alto, Calif. Other reagents were analytical grade.

Black-tailed prairie dogs (Cynomys ludovicianus) of either sex, weighing $0.75-1.0 \mathrm{~kg}$ were obtained from Otto M. Locke, New Braunfels, Tex. These animals were fed a cholesterolfree diet (guinea pig chow supplemented with corn) for at least 3 mo before our studies in order to avoid inadvertent cholesterol ingestion. The animals were kept in stainless steel wire cages at a temperature of $23.0 \pm 1.0^{\circ} \mathrm{C}$ and fed Purina lab chow (Ralston Purina Co., St. Louis, Mo.) and water ad lib., except as indicated.

Organ culture. 'Prairie dog gallbladder, gastric and colonic explants were maintained in organ culture for up to $24 \mathrm{~h}$ by the following modification of the method of Browning and Trier (28). Explants (1.5 $\mathrm{mm}^{2}$, three per dish) were cultured in triplicate in Trowell's T8 medium without fetal calf serum with $\left[{ }^{3} \mathrm{H}\right]$ glucosamine and added unlabeled glucosamine to give a final concentration of $1.0 \mathrm{mM}$ unless otherwise indicated. In some experiments, gallbladder explants were incubated with $10 \mu \mathrm{Ci} / \mathrm{ml}$ of $\left[{ }^{3} \mathrm{H}\right]$ mannose. After 4,10 , and $24 \mathrm{~h}$ of incubation, the incorporation of radioisotope into tissue and secreted (medium) glycoproteins was measured after trichloroacetic acid precipitation, as described by LaMont and Ventola (29). Endogeneous concentration of glucosamine of gallbladder explants from control and cholesterol-fed animals was determined using the method of Forsdyke (30) as modified by Alpers and Philpott (31). Histological examination of explants incubated for $24 \mathrm{~h}$ (courtesy of J. S. Trier) revealed excellent morphologic preservation.

Paper chromatography. To determine the chemical identity of incorporated radioactivity, radiolabeled tissue and 
secreted glycoproteins from gallbladder organ culture experiments (24-h incubations) were precipitated as described above. These precipitates were then acid hydrolyzed and the products subjected to paper chromatography (29). Greater than $95 \%$ of the radiolabeled hydrolytic products comigrated with authentic glucosamine or galactosamine.

Sepharose $4 B$ chromatography. $2 \mathrm{ml}$ of organ culture medium containing radiolabeled glycoproteins was exhaustively dialyzed against distilled water at $4^{\circ} \mathrm{C}$ for $72 \mathrm{~h}$ and loaded on a $10 \times 1.6-\mathrm{cm}$ column of Sepharose $4 \mathrm{~B}$ equilibrated with $10 \mathrm{mM}$ sodium phosphate buffer, $\mathrm{pH}$ 7.4. The column was eluted with the same buffer at a rate of 16 $\mathrm{ml} / \mathrm{h}$. Radioactivity was monitored in each $1-\mathrm{ml}$ fraction by counting an aliquot in $10 \mathrm{ml}$ of Redisolv-HP. The elution of radiolabeled glycoproteins was compared with the elution of the following standards: blue dextran $(2,000,000$ mol wt) ovalbumin $(45,000 \mathrm{~mol} \mathrm{wt})$ and vitamin $B_{12}(1,557$ mol wt). Uronic acid analysis on the void volume peak was performed colorimetrically (32).

Collection of bile. Gallbladder and hepatic bile was collected from prairie dogs after a 12-h fast. The animals were lightly anesthetized with intraperitoneal pentothal (Thiopentol sodium, Abbott Diagnostics, North Chicago, Ill.), and the upper abdomen was opened. The cystic duct was doubly ligated and gallbladder bile was collected by aspiration with an 18-gauge needle and syringe prewarmed to $37^{\circ} \mathrm{C}$. After cholecystectomy, the common bile duct was cannulated and $2 \mathrm{ml}$ of hepatic bile was collected after discarding the first $0.25 \mathrm{ml}$ to avoid contamination from gallbladder bile. Bile samples were processed by two methods. In one, aliquots of uncentrifuged bile were frozen at $-20^{\circ} \mathrm{C}$ and used for total and relative lipid analysis after thawing and homogenization. This is referred to as "homogenized" bile, which may, in addition to micellar lipids, contain cholesterol liquid and solid crystals and small cholesterol stones. In the second method, the bile sample was first centrifuged $(10,000 \mathrm{~g})$ for $2 \mathrm{~min}$ at $37^{\circ} \mathrm{C}$ in a Beckman microfuge (Beckman Instruments, Inc.), and the clear supernatant micellar phase was separated by aspiration with a Pasteur pipette. This bile contained only a single aqueous phase (micellar phase) and is hereafter referred to as "separated" bile.

Direct and polarizing microscopy. Fresh bile samples maintained at $37.0 \pm 1.5^{\circ} \mathrm{C}$ with a Mettler (FP 5; Mettler Instrument Corp., Hightstown, N. J.) heating stage were examined for crystals, liquid crystals, stones, and mucus gel by direct and polarizing light microscopy (Photomicroscope III, Carl Zeiss, Inc., New York). Cholesterol monohydrate crystals were identified by birefrigence and typical notched rhomboidal shapes (33). Liquid crystals containing cholesterol and lecithin were identified as birefringent Maltese crosses (focal conics) and by their compressibility (2). Mucus gel appeared as nonbirefringent amorphous strands.

Nucleation experiments. Gallbladder epithelial mucin was prepared from human gallbladder specimens obtained at operation from patients undergoing cholecystectomy for cholelithiasis. Gallbladder mucosal strips, $\sim 2 \times 2 \mathrm{~cm}$, were rinsed free of blood and debris in iced saline, then scraped with the edge of a glass microscope slide to remove the mucosa. The mucosal scrapings were then suspended in $10 \mathrm{vol}$ of $10 \mathrm{mM}$ Tris buffer, pH 8.0 , and homogenized in a Waring blender at high speed for $30 \mathrm{~s}$. The homogenate was centrifuged at $100,000 \mathrm{~g}$ for $60 \mathrm{~min}$, and the supernate lyophilized. Before gel filtration, the lyophilized supernate was resuspended in Tris buffer and loaded onto a $100 \times 2.4-\mathrm{cm}$ column of Sepharose 4B. The column was eluted with Tris buffer at a rate of $20 \mathrm{ml} / \mathrm{h}$, and the void volume fractions containing high molecular weight mucin were pooled, dialyzed, and lyophilized. The lyophilized mucin was added to hepatic bile obtained from prairie dogs fed cholesterol for $14 \mathrm{~d}$, to give a final mucin concentration of $20 \mathrm{mg} / \mathrm{ml}$. At this concentration the mucin formed a translucent gel similar to that observed in the gallbladder after $5 \mathrm{~d}$ or more of cholesterol feeding. The bile and mucin were thoroughly mixed in the well of a microscope slide and observed by polarizing microscopy at $37^{\circ} \mathrm{C}$ for crystal as described above. Control slides contained mucin alone, suspended in saline; the same bile mixed with bovine serum albumin at $20 \mathrm{mg} / \mathrm{ml}$ final concentration; or the same bile without any additions. The samples were observed hourly for the first $6 \mathrm{~h}$ and every $6 \mathrm{~h}$ thereafter up to $24 \mathrm{~h}$. This experiment was performed on three separate specimens of gallbladder mucin.

Lipid analysis. Biliary phospholipids were measured as inorganic phosphorus by the method of Bartlett (34). Cholesterol was determined with Carr and Drekter's (35) modification of the original method of Abell et al. (36). Total bile salt concentration was measured enzymatically by the 3- $\alpha$ hydroxysteroid dehydrogenase method of Talalay (37) as modified by Admirand and Small (38). Biliary sterols were extracted with hexane from samples of gallbladder bile $(0.1 \mathrm{ml}$ bile in $0.1 \mathrm{ml}$ hexane) and gallstones ( $1.0 \mathrm{mg}$ crushed stone powder in $1.0 \mathrm{ml}$ hexane) as well as from control and lithogenic diet ( $10 \mathrm{~g}$ chow in $0.5 \mathrm{ml}$ hexane). These mixtures were shaken vigorously at room temperature for 10 min, and the hexane phase was analyzed for cholesterol and other sterols by gas-liquid chromatography by the method of Miettinen et al. (39). Cholesterol and $5 \alpha$-cholestanol were used as external standards. We analyzed the bile acids of gallbladder samples from control and cholesterol-fed animals using high performance liquid chromatography (Altex model $110 \mathrm{~A}$, Beckman Instruments, Inc.) The mobile phase was $75 \%$ methanol in aqueous $0.005 \mathrm{M}$ $\mathrm{KH}_{2} \mathrm{PO}_{4}$ at $\mathrm{pH} 5.0$ at a pressure of $3,000 \mathrm{psi}$ through a reversed-phase octadecylsilane column. The detector system was a variable wavelength UV-monitor set at $210 \mathrm{~nm}$, recorded on an Altex C-RIA integrator-recorder. We plotted the relative lipid compositions on triangular coordinates using phase boundary limits for different total lipid concentrations generated by Carey and Small (2). Saturation indices of hepatic and gallbladder bile samples were calculated individually from the critical tables of Carey (40), which corrects the saturation index of each bile sample for its own total lipid concentration.

Preparation of artificial mixed micelles. Mixed micellar solutions containing sodium taurocholate, cholesterol $(1,3,5$, or $7 \mathrm{~mol} / 100 \mathrm{~mol}$ ), and lecithin were prepared by coprecipitation from methanol or ethanol as described (2). The relative and total lipid concentrations were adjusted to approximate those of prairie dog gallbladder bile (total lipid concentration $10 \mathrm{~g} / \mathrm{dl}$; molar bile salt to lecithin ratio, 8.5: 1.5). The lipids were lyophilized and reconstituted to the desired total lipid concentration by addition of organ culture medium.

Preparation of lithogenic diet. Recrystallized cholesterol was dissolved in absolute ethanol, thoroughly mixed with Purina chow pellets (Ralston Purina) at a final concentration of $1.2 \%$ cholesterol by weight, then dried. To prevent auto-oxidation of cholesterol in chow during storage, we prepared fresh chow every $4 \mathrm{~d}$.

Statistical method. Control and experimental data were compared using Student's two-tailed unpaired $t$ test.

\section{Experimental design}

Induction of cholesterol gallstones. Prairie dogs were fed a $1.2 \%$ cholesterol diet and sacrificed for study at $3,5,7$, and 
$14 \mathrm{~d}$ after commencement of feeding. Control animals were fed identical chow without cholesterol. At each time point, gallbladder contents and hepatic biles were collected and studied by direct and polarized light microscopy, and analyzed for lipids; the gallbladders were then removed for organ culture studies.

Withdrawal of lithogenic diet. To determine the effect of withdrawal of cholesterol from the diet on gallbladder glycoprotein synthesis in organ culture, animals were first fed the lithogenic diet for $5 \mathrm{~d}$. The lithogenic diet was then stopped and the animals were refed the control chow. Gallbladder glycoprotein synthesis and secretion were measured in organ culture 3 and $5 \mathrm{~d}$ after resumption of the regular diet.

Cystic duct ligation. To determine the effect of exclusion of the gallbladder from the biliary tree on gallbladder glycoprotein synthesis and secretion, we ligated the cystic duct of animals before cholesterol feeding. Under light pentothal anesthesia, the gallbladders of unfed prairie dogs were milked gently to empty the contents into the common bile duct. The cystic duct and cystic artery were carefully dissected with the aid of a magnifying lens. The cystic duct was doubly ligated with 5-0 silk, care being taken to avoid injury to the cystic artery. The abdominal incision was then closed in layers. Sham-operated animals were treated identically, except that the cystic duct was isolated but not ligated. After a 4-d recovery period, the animals were fed the lithogenic diet for $7 \mathrm{~d}$. Glycoprotein synthesis and secretion in organ culture was measured in cystic ductligated and sham-operated controls on the 8th $\mathrm{d}$ of cholesterol feeding (12th d postoperative).

Effect of artificial mixed micelles and supersaturated hepatic bile on in vitro glycoprotein synthesis and secretion. We measured glycoprotein synthesis and secretion in gallbladder explants from control (noncholesterol-fed) animals after incubation with media containing either unsaturated or supersaturated artificial mixed micelles, or supersaturated hepatic bile from 5-d cholesterol-fed animals. In one set of experiments, the explants were preincubated for $30 \mathrm{~min}$ at $37^{\circ} \mathrm{C}$ with organ culture medium containing the mixed micellar solutions. The explants were then thoroughly washed three times with fresh organ culture medium and cultured for an additional $24 \mathrm{~h}$ in medium containing $\left[{ }^{3} \mathrm{H}\right]$ glucosamine without micellar solutions. In another series of experiments, the gallbladder explants were incubated continuously with mixed micellar solutions in culture medium containing $\left[{ }^{3} \mathrm{H}\right]$ glucosamine for $24 \mathrm{~h}$. The incorporation of $\left[{ }^{3} \mathrm{H}\right]$ glucosamine into tissue and secreted glycoproteins was determined as described above.

To determine the effect of native supersaturated bile on glycoprotein synthesis of control gallbladders in vitro, hepatic bile was collected from prairie dogs fed the lithogenic diet for $5 \mathrm{~d}$, and lyophilized in $0.75-\mathrm{ml}$ aliquots after centrifugation. The dried bile was then reconstituted with $0.75 \mathrm{ml}$ organ culture medium to achieve the original total lipid concentration and incubated continuously with control prairie dog gallbladder for $24 \mathrm{~h}$. Glycoprotein synthesis and secretion were then quantified as described.

\section{RESULTS}

General effects of the lithogenic diet: gross and microscopic observations of bile and gallbladder. Cholesterol-enriched chow was readily eaten by prairie dogs, in amounts comparable to that of control chow. No weight loss or other sign of illness was observed in any animal during cholesterol feeding. Hepatic bile from both control $(n=12)$ and cholesterol-fed animals $(n=31)$ was, without exception, grossly and microscopically clear. Gallbladder bile from control animals was always clear, without gross or microscopic evidence of mucus gel, liquid crystals, cholesterol monohydrate crystals, or stones; and gallbladder mucosa was smooth and glistening. The gallbladder bile after $3 \mathrm{~d}$ of feeding the lithogenic diet $(n=6)$ was indistinguishable grossly and microscopically from control bile. At $5 \mathrm{~d}$ of feeding $(n=8)$, the gallbladder bile was grossly turbid. Microscopy showed this turbidity to be due to mucus threads, cholesterol monohydrate crystals, and, in many cases, liquid crystals, but not to stones. Gross examination of the gallbladder wall at $5 \mathrm{~d}$ revealed a layer of adherent mucus gel in which numerous cholesterol monohydrate crystals were embedded. By $8 \mathrm{~d}$ of cholesterol feeding $(n=6)$, the turbidity of gallbladder bile was more pronounced, with a thicker layer of mucus and more cholesterol crystals. At day $14(n=11)$, the gallbladders were somewhat shrunken and in 5 of 11 animals, no liquid bile could be aspirated through an 18-gauge needle. When opened, these gallbladders revealed a very thick mucus gel that contained numerous $1-2-\mathrm{mm}$ yellowish cholesterol gallstones. Polarizing microscopy revealed the presence of mucus gel, numerous cholesterol crystals, and gallstones. In two animals, the stones had migrated into the cystic duct, but no animal had stones in the common duct.

Histologic sections of the gallbladder wall from cholesterol-fed animals when compared with controls showed no evidence of inflammation of the gallbladder during cholesterol feeding. Aerobic and anaerobic cultures of 14-d gallbladder bile $(n=6)$ were sterile.

Effect of glucosamine concentration on glycoprotein synthesis. The rate of $\left[{ }^{3} \mathrm{H}\right]$ glucosamine incorporation into gallbladder epithelial glycoproteins is dependent in part upon substrate (glucosamine) concentration in the organ culture medium. We therefore performed a dose-response study comparing $\left[{ }^{3} \mathrm{H}\right]$ glucosamine incorporation with total glucosamine concentration in gallbladders from controls and animals fed cholesterol for $8 \mathrm{~d}$ (Fig. 1). The dose-response relationship in both control and cholesterol-fed animals was described by sigmoid curves, with the half maximal glucosamine concentration at $\sim 1 \mathrm{mM}$ in both. Glucosamine concentrations $>5 \mathrm{mM}$ were mildly toxic to gallbladder explants, as evidenced by pyknotic nuclei and karyolysis on light microscopy. Higher concentrations of glucosamine have been shown to inhibit protein, RNA, and DNA biosynthesis in normal and neoplastic tissues (41). We therefore performed all incubations at $1 \mathrm{mM}$ glucosamine concentration to avoid toxicity.

Influence of incubation time on gallbladder glyco- 


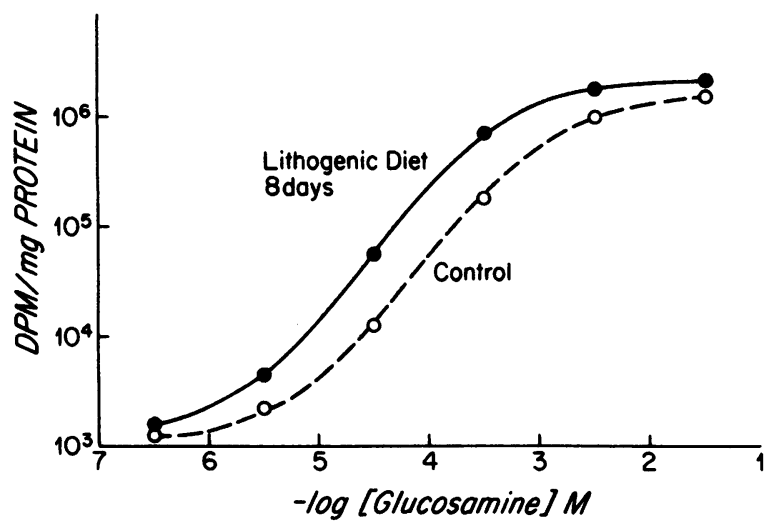

Figure 1 24-h organ culture of gallbladder explants. Doseresponse relationship between glucosamine concentration and glycoprotein synthesis by explants from animals fed control chow (O) and $1.2 \%$ cholesterol chow for $8 \mathrm{~d}(\Theta)$.

protein synthesis and secretion. $\left[{ }^{3} \mathrm{H}\right] \mathrm{Glucosamine}$ was incorporated into gallbladder tissue and secreted glycoproteins in a progressive fashion at 4,10 , and $24 \mathrm{~h}$ of incubation (Table I). The total (tissue plus secreted) incorporation into glycoproteins at each time point was greater in the 5-d lithogenic diet animals than in the controls. This increase was particularly striking after 10 and $24 \mathrm{~h}$ of incubation. In addition, cholesterol feeding was associated with a change in the proportion of $\left[{ }^{3} \mathrm{H}\right]$ glucosamine incorporated into secreted vs. tissue glycoproteins. In control animals, the percentage of radioactivity in secreted glycoproteins accounted for $24 \%$ of total radioactivity at 4 and $10 \mathrm{~h}$, and $32 \%$ at $24 \mathrm{~h}$. In cholesterol-fed animals, secreted glycoproteins accounted for $33 \%$ of radioactivity at $4 \mathrm{~h}, 50 \%$ at $10 \mathrm{~h}$, and $49 \%$ at $24 \mathrm{~h}$.

Influence of duration of lithogenic diet on gallbladder glycoprotein synthesis and secretion. Cholesterol feeding produced an increase in glycoprotein synthesis and secretion at $3,5,8$, and $14 \mathrm{~d}$, and after 4, 10, and $24 \mathrm{~h}$ incubation when compared with control values (Table I). These differences were significant at all times studied except the 24-h secretion value on day 3 . The greatest increases were observed on the 5th $\mathrm{d}$ of cholesterol feeding. For purposes of illustration, the 10-h incubation data at $3,5,8$, and $14 \mathrm{~d}$ of cholesterol diet are shown in Fig. 2. The mean increase in secretion of $\left[{ }^{3} \mathrm{H}\right]$ glucosamine-labeled glycoproteins was $516 \%$ of control on day 5 , while the corresponding increase in incorporation of $\left[{ }^{3} \mathrm{H}\right]$ glucosamine into gallbladder tissue was $116 \%$ of control. The rate of incorporation into both compartments fell dramatically at 8 and $14 \mathrm{~d}$, but was still significantly higher than control.

To determine whether cholesterol feeding influenced the synthesis of membrane-type glycoproteins in the gallbladder as well as mucin-type glycoproteins, we measured the incorporation of $\left[{ }^{3} \mathrm{H}\right]$ mannose into gallbladder explants and medium glycoproteins. Mannose is commonly found in membrane glycoproteins but is not a constituent of mucin glycoproteins (42). The incorporation of $\left[{ }^{3} \mathrm{H}\right]$ mannose into tissue glycoproteins of gallbladder explants from prairie dogs fed

TABLE I

Organ Culture Studies: Influence of Cholesterol Feeding on Incorporation of $\left[{ }^{3} \mathrm{H}\right]$ Glucosamine into Gallbladder Glycoproteins

\begin{tabular}{|c|c|c|c|c|c|}
\hline \multirow[b]{2}{*}{ Incubation time } & \multirow[b]{2}{*}{$\begin{array}{l}\text { Control } \\
(n=12)\end{array}$} & \multicolumn{4}{|c|}{ Number of days on lithogenic diet } \\
\hline & & $\begin{array}{c}3 \\
(n=5)\end{array}$ & $\begin{array}{c}5 \\
(n=8)\end{array}$ & $\begin{array}{c}8 \\
(n=5)\end{array}$ & $\begin{array}{c}14 \\
(n=5)\end{array}$ \\
\hline$h$ & & \multicolumn{2}{|c|}{$D P M / m g$} & \multicolumn{2}{|c|}{$D P M / m g$} \\
\hline \multicolumn{6}{|c|}{ Tissue glycoproteins } \\
\hline 4 & $14.2 \pm 0.3$ & $21.0 \pm 1.9^{*}$ & $27.0 \pm 4.4 \ddagger$ & $25.5 \pm 3.5 \S$ & $24.0 \pm 4.1 \S$ \\
\hline 10 & $38.1 \pm 2.9$ & $47.5 \pm 4.1^{*}$ & $82.5 \pm 5.4 \ddagger$ & $57.5 \pm 7.9 \S$ & $56.0 \pm 4.4 \S$ \\
\hline 24 & $78.4 \pm 1.3$ & $98.5 \pm 8.5^{*}$ & $146.5 \pm 6.0 \ddagger$ & $105.0 \pm 10.1 \S$ & $120.0 \pm 6.4 \oint$ \\
\hline \multicolumn{6}{|c|}{ Secreted glycoproteins } \\
\hline 4 & $4.4 \pm 0.5$ & $8.4 \pm 1.3^{*}$ & $13.5 \pm 1.6 \ddagger$ & $8.9 \pm 0.9 \S$ & $8.7 \pm 1.4 \oint$ \\
\hline 10 & $11.8 \pm 0.9$ & $18.1 \pm 1.7^{*}$ & $70.9 \pm 5.8 \ddagger$ & $37.0 \pm 2.9 \S$ & $28.9 \pm 2.8 \S$ \\
\hline 24 & $37.4 \pm 5.9$ & $41.9 \pm 2.8$ & $138.6 \pm 19.9 \ddagger$ & $70.2 \pm 3.0 \S$ & $59.3 \pm 3.5 \S$ \\
\hline
\end{tabular}

Results are expressed as DPM/mg protein $\times 10^{-4}$ (mean \pm SEM). The figures in parentheses are the numbers of animals studied. Explants of gallbladders were incubated with [ ${ }^{3} \mathrm{H}$ ]glucosamine for the times indicated. Incorporation of radiolabel into tissue and secreted glycoproteins was measured as described in Methods. Each assay was performed in triplicate.

${ }^{*} P<0.05$, compared with control value at same incubation time.

$\$ P<0.001$, compared with control value at same incubation time.

$\S P<0.01$, compared with control value at same incubation time. 


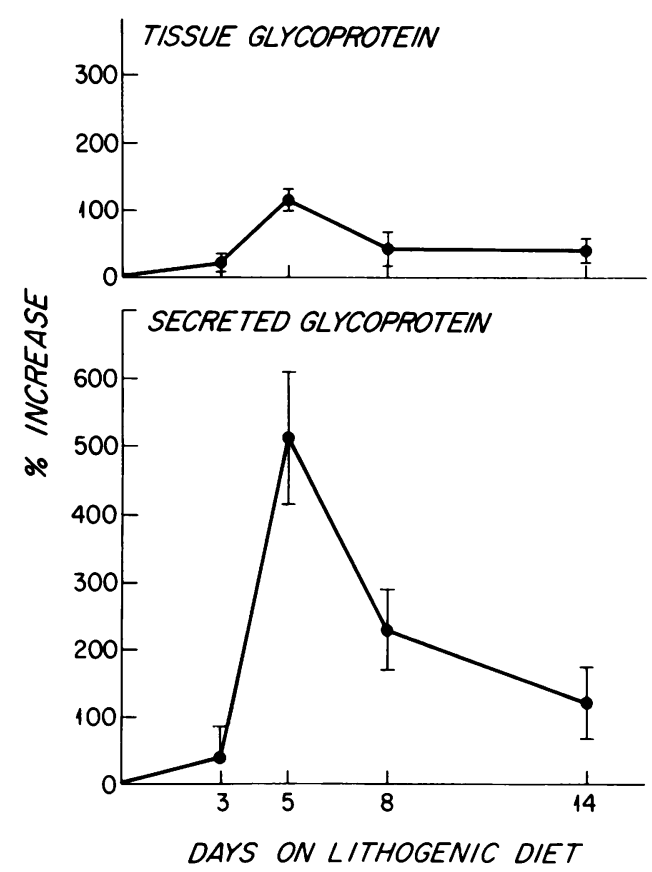

FIGURE 2 Influence of duration of lithogenic diet on the incorporation of $\left[{ }^{3} \mathrm{H}\right]$ glucosamine into tissue and secreted glycoproteins. The vertical axis represents percent increase compared with control values. Each point represents the mean percentage increase $\pm S E M$ compared to control.

cholesterol for $5 \mathrm{~d}$ was $102 \%$ of control $(n=14 \mathrm{ex}$ plants in each group from two separate experiments, $P>0.1$ ). Organ culture medium from both cholesterol-fed and control gallbladders did not contain significant protein-bound radioactivity, which confirms that mannose is not incorporated into secreted mucin glycoproteins.

Influence of lithogenic diet on endogenous glucosamine pool size. To test whether the dramatic increases in glycoprotein production were artifacts of a change in endogenous glucosamine concentration induced by cholesterol feeding, we estimated the pool size of glucosamine in gallbladder explants from control and cholesterol-fed (5-d lithogenic diet) animals. In Fig. 3 we plot the concentration of added unlabeled glucosamine vs. the reciprocal of incorporated $\left[{ }^{3} \mathrm{H}\right]$ glucosamine after $10 \mathrm{~h}$ of incubation. Despite the mild morphologic alterations observed at higher substrate concentrations, the incorporation of glucosamine into gallbladder explants was proportional to dose. The data points fell on two straight lines, whose slopes reflect the rates of glycoprotein synthesis; that is, the greater the rate of synthesis, the steeper the slope. The linear regression equations indicate that on the lithogenic diet, tissue glycoprotein incorporation is 2.9 -fold that of control, a value which corresponds to our earlier data (Table I).

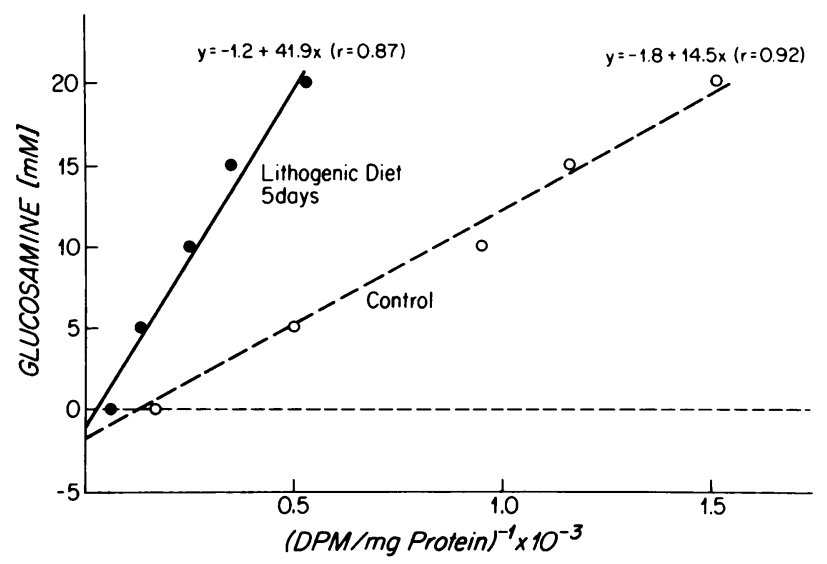

Figure 3 Influence of 5-d lithogenic and control diets on endogenous glucosamine concentration in gallbladder explants, determined by the isotope dilution technique (see Methods). Glucosamine concentrations were obtained by extrapolation of the linear regression to the $y$-axis.

The vertical intercepts of these lines give the endogenous concentrations of glucosamine, which were $1.8 \mathrm{mM}$ for control and $1.2 \mathrm{mM}$ in the lithogenic diet-fed animals. This small difference is unlikely to account for the marked increase in glycoprotein synthesis and secretion in cholesterol-fed animals.

Influence of lithogenic diet on glycoprotein synthesis and secretion in other organs. Increased dietary cholesterol might be expected to affect glycoprotein synthesis and secretion in the mucosa of the stomach or colon since both epithelia are in direct contact with excess cholesterol in food and feces. To determine whether cholesterol feeding caused an increase in mucin synthesis and secretion in these organs, we measured the incorporation of $\left[{ }^{3} \mathrm{H}\right]-$ glucosamine into explants of glandular stomach and colon from control and 5-d cholesterol-fed prairie dogs. Preliminary experiments indicated that gastric mucosal explants underwent autolysis after $8 \mathrm{~h}$ incubation, but were normal by light microscopy after $4 \mathrm{~h}$ incubation. Incorporation of $\left[{ }^{3} \mathrm{H}\right]$ glucosamine $(4 \mathrm{~h})$ into explant glycoproteins of 5-d cholesterol-fed explants was $100.5 \%$ of control, and for secreted glycoproteins was $99.6 \%$ of control $(n=14$ determinations from two separate experiments, $P>0.1$ ). Similarly, the incorporation of $\left[{ }^{3} \mathrm{H}\right]$ glucosamine into colonic explants from 5-d cholesterol-fed animals was $96.6 \%$ of control, and into secreted colonic glycoproteins was $108.6 \%$ of control $(P>0.1)$. Thus the stimulatory effect of cholesterol feeding on mucus secretion appeared to be specific to the gallbladder.

Sepharose $4 B$ chromatography of secreted glycoproteins. To determine that the incorporated radioactivity in secreted glycoproteins was in fact due to a high molecular weight mucus glycoprotein rather than 
a shed membrane glycoprotein, we performed gel chromatography (Sepharose 4B) of dialyzed organ culture medium after $24 \mathrm{~h}$ incubation with gallbladder explants from control and cholesterol-fed animals. Approximately $85 \%$ of macromolecular radioactivity eluted in the void volume, which indicates a glycoprotein with an approximate molecular weight of at least $1 \times 10^{6}$. A smaller radiolabeled glycoprotein comprising $\sim 15 \%$ of the total radioactivity eluted in the included volume near the peak fraction of ovalbumin $(45,000 \mathrm{~mol} \mathrm{wt})$. The elution patterns from Sepharose $4 \mathrm{~B}$ of secreted glycoproteins from the gallbladders of cholesterol-fed animals were essentially the same as that seen in control animals. Intestinal (43) and colonic (44) mucins have previously been shown to elute in the void volume of Sepharose 4B. Uronic acid as determined colorimetrically was not present in these peak fractions, which indicates the absence of radiolabeled glycosaminoglycans, which would also be expected to elute in the void volume of this column.

Relative lipid compositions, identification of insoluble phases, and lithogenic indices of hepatic and gallbladder biles. In Fig. 4 we plot on triangular coordinates the relative lipid compositions of homogenized hepatic and gallbladder biles. In addition, we plot the micellar phase boundaries (cholesterol-solubility limits) which correspond to the upper and lower total lipid concentrations in each set of biles $(2,40)$. As expected, a progressive increase in the relative cholesterol concentration of hepatic and gallbladder biles occurred with cholesterol feeding. Both hepatic and gallbladder bile reached saturation on the 5 th $d$, and were distinctly supersaturated by 8 and $14 \mathrm{~d}$. As in other experimental models and in man, when bile becomes more enriched in cholesterol, the phospholipid to bile salt ratio also increases (45). In Fig. 5 we plot the mean relative lipid compositions of hepatic and gallbladder bile shown in Fig. 4. The micellar phase boundary in the figure is drawn according to the mean total lipid concentration in each set of biles. This clearly shows the progressive shift upward and to the right in relative biliary lipid compositions. This shift corresponds to an increase in cholesterol content, a relative decrease in total bile salt content, and a relative increase in phospholipid content. As indicated in Fig. 5, hepatic biles were always a one-phase micellar system, whereas gallbladder bile at 5,8 , and $14 \mathrm{~d}$ consisted of more than one phase, namely micellar bile, and liquid and solid cholesterol crystals. To determine the relative lipid concentration of the micellar phases isolated from these samples, we prepared and analyzed separated bile as described in Methods.

In Fig. 6 we compare the saturation indices of homogenized and separated bile. Hepatic bile from control and experimental animals was always a onephase system, and, therefore, the saturation indices of both homogenized and separated bile are similar. Similarly, gallbladder bile on day 5 showed similar saturation indices in homogenized and centrifuged samples. In contrast, 8-d homogenized gallbladder bile was apparently far more saturated than separated gallbladder bile. This difference can be attributed to the inclusion of liquid and solid crystals of cholesterol in homogenized bile and to their removal in centrifuged bile. In addition, the plot reveals that centrifuged gallbladder bile remained just saturated at 5 and $8 \mathrm{~d}$, whereas the saturation indices of hepatic bile increased between these two time points.

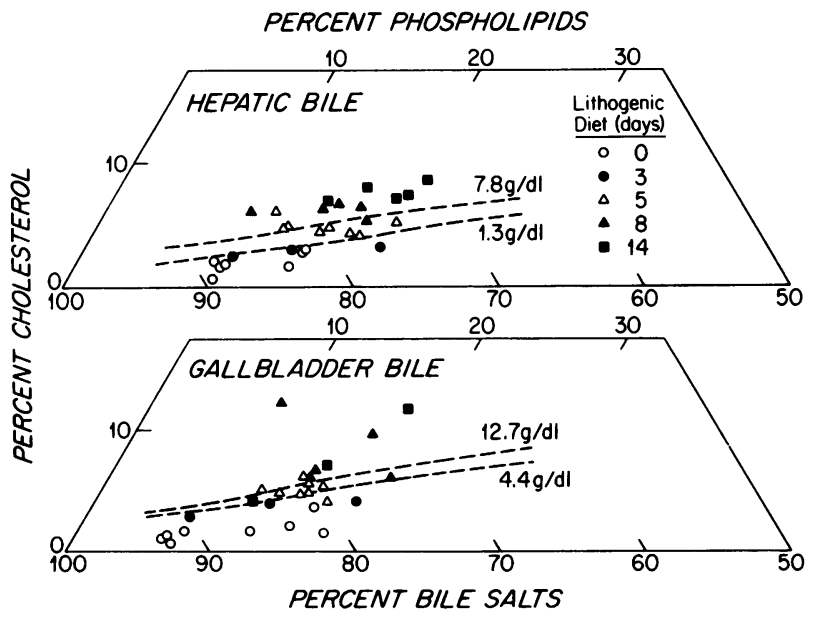

FIgURE 4 Triangular coordinate plots of the relative lipid composition of homogenized hepatic and gallbladder biles of control and lithogenic diet-fed animals. The dashed lines represent the cholesterol solubility limits for the indicated ranges of total lipid concentrations.

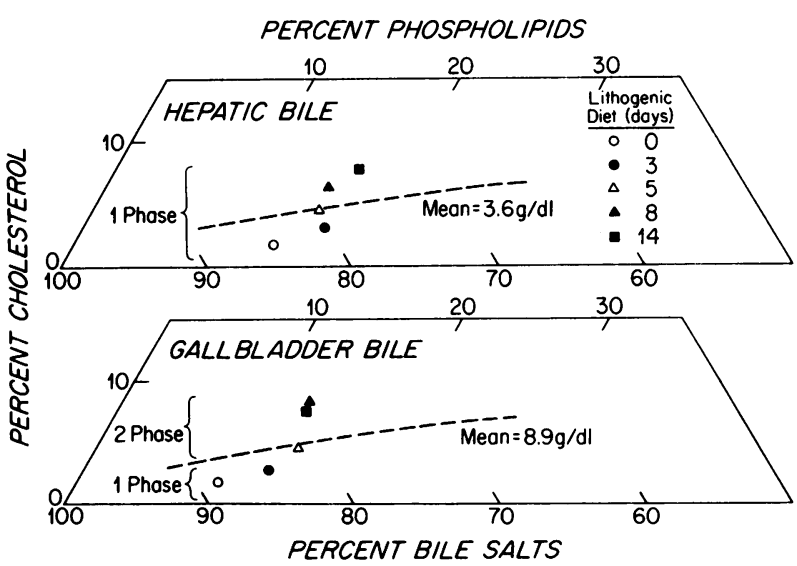

Figure 5 Triangular coordinate plots of the mean relative lipid compositions and number of phases present in hepatic and gallbladder biles of control and lithogenic diet-fed animals. The phase boundaries are drawn according to the mean total lipid concentrations in both hepatic and gallbladder biles. 


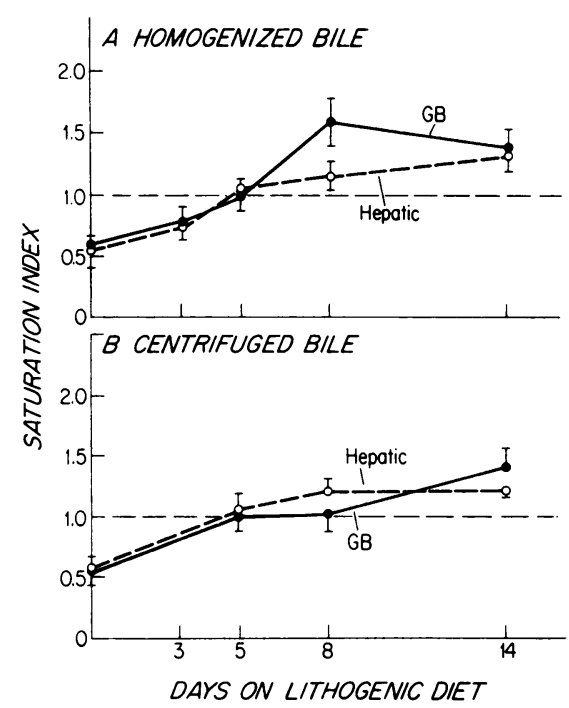

Figure 6 Cholesterol-saturation indices of (A) homogenized and $(B)$ separated gallbladder bile $(O)$ and hepatic bile $(O)$ of control and during lithogenic diet feeding. Mean \pm SEM compositions are plotted vs. the number of days on the lithogenic diet. The saturation index of each bile sample was calculated after correcting for its total lipid concentration.

Nevertheless, by $14 \mathrm{~d}$ centrifuged gallbladder bile became clearly supersaturated.

Analysis of individual bile salts in gallbladder bile by high performance liquid chromatography revealed that all bile acids were taurine conjugated. Taurocholate comprised the major (range 91-94\%) bile salt in both control and lithogenic bile; taurochenodeoxycholate (5.4-6.1), taurodeoxycholate $(0.1-1.3)$, and taurolithocholate $(0.2-1.0)$ were present in much smaller concentrations. The only difference we observed in gallbladder bile during cholesterol feeding was a marked decrease in the concentration of secondary bile salts, taurodeoxycholate, and taurolithocholate at $14 \mathrm{~d}$. We did not observe any unusual bile salts which might cause mucus hypersecretion.

To verify that cholesterol without appreciable quantities of cholesterol oxidation products $(46,47)$ was being fed to our animals, we analyzed by gas-liquid chromatography the regular and cholesterol-treated chow, as well as gallbladder bile and gallstones of cholesterol-fed animals. The control prairie dog chow contained only a trace $(0.8 \%)$ of cholesterol. The lithogenic diet contained a major cholesterol peak, but no significant cholesterol oxidation products. Cholesterol constituted $94.7 \%$ of the sterols in gallbladder bile ( $5 \mathrm{~d}$ of cholesterol feeding) and $90.4 \%$ of the sterols extracted from gallstones. To summarize, qualitative lipid analysis of bile revealed no major changes in the types of bile acids, and no evidence for any unusual sterols.

Effect of withdrawal of lithogenic diet on gallbladder glycoprotein synthesis. To determine whether the increased glycoprotein synthesis would return to normal after cholesterol feeding was terminated, we fed prairie dogs the lithogenic diet for $5 \mathrm{~d}$, then abruptly withdrew the diet and placed the animals on regular chow for $5 \mathrm{~d}$. From the peak level at $5 \mathrm{~d}$ on the lithogenic diet, the incorporation of $\left[{ }^{3} \mathrm{H}\right]-$ glucosamine into gallbladder glycoproteins declined significantly $3 \mathrm{~d}$ after stopping the diet and was normal $5 \mathrm{~d}$ after withdrawal of the cholesterol diet (Table II). These results indicate that the increased synthesis of glycoprotein observed during cholesterol feeding is dependent upon continuation of cholesterol feeding.

Effect of cystic duct ligation on glycoprotein synthesis. Since previous studies by Freston et al. (18) in rabbits indicated that cystic duct ligation prevented mucus accumulation in the gallbladder of rabbits fed $5 \alpha$-cholestanol, we determined the effect of cystic duct ligation on glycoprotein synthesis and secretion of cholesterol-fed prairie dogs. Cystic duct ligation resulted in marked diminution of gallbladder glycoprotein synthesis and secretion in cholesterol-fed prairie dogs compared with sham-operated cholesterol-fed controls (four animals in each group). Total glycoprotein synthesis (tissue plus secreted) was reduced $45.1 \%(P<0.01)$ at $24 \mathrm{~h}$ of incubation in explants from cystic duct-ligated animals when compared with cholesterol-fed controls. Total glycoprotein synthesis in the cystic duct-ligated group did not differ significantly from animals fed the control diet. These results suggest that the stimulus to increased gallbladder glycoprotein synthesis is carried to the gallbladder via bile. Cystic duct ligation produced neither necrosis nor atrophy of the gallbladder, which makes it unlikely that the results observed were secondary to nonspecific tissue damage.

Effect of artificial bile and reconstituted prairie dog lithogenic bile on glycoprotein synthesis. We attempted to determine whether incubation of control gallbladder explants with saturated bile caused increased glycoprotein synthesis. In the first experiment, we preincubated normal gallbladder explants for 60 min in artificial bile before transferring them to fresh culture medium with $\left[{ }^{3} \mathrm{H}\right]$ glucosamine for a 24-h incubation. Increasing concentrations of cholesterol had no effect on glycoprotein synthesis. In a second experiment, gallbladder explants from control animals were incubated continuously for $24 \mathrm{~h}$ with artificial bile. This likewise had no effect on the rate of glycoprotein synthesis. Similar negative results were obtained with native bile from cholesterol-fed animals.

Nucleation of bile by gallbladder mucin gel. Gallbladder mucin glycoproteins were prepared from human gallbladder mucosa obtained from elective cholecystectomy specimens. Three separate mucin preparations were studied in regard to their ability to cause nucleation of saturated bile. Mucin at a concentration of $20 \mathrm{mg} / \mathrm{ml}$ was mixed with saturated 
TABLE II

Effect of Withdrawal of Lithogenic Diet on Incorporation of

$\left[{ }^{3} \mathrm{H}\right]$ Glucosamine into Gallbladder Glycoproteins

\begin{tabular}{|c|c|c|c|c|}
\hline \multirow[b]{2}{*}{ Incubation time } & \multirow{3}{*}{$\begin{array}{c}\text { Control } \\
(n=12)\end{array}$} & \multicolumn{3}{|c|}{ Lithogenic diet } \\
\hline & & & & $\begin{array}{c}\text { Fed } 5 \mathrm{~d}, \\
\text { discontinued }\end{array}$ \\
\hline \multirow[t]{2}{*}{$h$} & & $(n=8)$ & $(n=3)$ & $(n=3)$ \\
\hline & \multicolumn{4}{|c|}{$D P M / m g$} \\
\hline \multicolumn{5}{|c|}{ Tissue glycoproteins } \\
\hline 4 & $14.2 \pm 0.3$ & $27.0 \pm 4.4^{*}$ & $22.1 \pm 2.1 \ddagger$ & $16.1 \pm 1.3$ \\
\hline 10 & $38.1 \pm 2.9$ & $82.5 \pm 5.4^{*}$ & $45.8 \pm 3.7 \ddagger$ & $40.4 \pm 3.2$ \\
\hline 24 & $78.4 \pm 1.3$ & $146.5 \pm 6.0^{*}$ & $97.4 \pm 8.1 \ddagger$ & $80.5 \pm 5.9$ \\
\hline \multicolumn{5}{|c|}{ Secreted glycoproteins } \\
\hline 4 & $4.4 \pm 0.5$ & $13.5 \pm 1.6^{*}$ & $7.7 \pm 2.1 \neq$ & $4.9 \pm 1.3$ \\
\hline 10 & $11.8 \pm 0.9$ & $70.9 \pm 5.8^{*}$ & $16.3 \pm 1.8 \ddagger$ & $12.2 \pm 2.2$ \\
\hline 24 & $37.4 \pm 5.9$ & $138.6 \pm 19.9^{*}$ & $40.1 \pm 3.6$ & $38.2 \pm 6.4$ \\
\hline
\end{tabular}

Incorporation of $\left[{ }^{3} \mathrm{H}\right]$ glucosamine was measured in organ culture of gallbladders from animals fed control; $5 \mathrm{~d}$ lithogenic; $5 \mathrm{~d}$ lithogenic, then $3 \mathrm{~d}$ control; and $5 \mathrm{~d}$ lithogenic, then 5-d control diets. Results are expressed as DPM/mg protein $\times 10^{-4}$.

${ }^{*} P<0.001$, compared with control value at same incubation time.

$\ddagger P<0.05$, compared with control value at same incubation time.

hepatic bile (saturation index 1.3) obtained from a prairie dog fed cholesterol for $14 \mathrm{~d}$. At this concentration, the mucin formed a viscous translucent gel. The results of a typical experiment are shown in Fig. 7. By polarizing-light microscopy, the hepatic bile and mucin gel mixture was noted to contain liquid crystals (Maltese crosses) after $2 \mathrm{~h}$ of incubation at $37^{\circ} \mathrm{C}$. At later time points $(18 \mathrm{~h})$, solid cholesterol crystals (notched rhombohedral plates) became visible, and were apparently nucleated from the liquid crystalline phase. Control incubations of hepatic bile mixed with $20 \mathrm{mg} / \mathrm{ml}$ bovine serum albumin, gallbladder mucin alone, or hepatic bile alone did not contain liquid or solid crystals even after $24 \mathrm{~h}$ incubation, at which time the experiments were terminated.

\section{DISCUSSION}

The initial observation in this study was that before the formation of cholesterol crystals there was visible accumulation of mucus gel in the gallbladder of prairie dogs fed a lithogenic diet. We then quantitated this effect by measuring the in vitro incorporation of $\left[{ }^{3} \mathrm{H}\right]$ glucosamine into gallbladder mucus glycoproteins in an organ culture system. This technique allowed us to maintain gallbladder explants in vitro for up to $24 \mathrm{~h}$ with excellent maintenance of morphologic detail, and to follow progressively at suitable time intervals the increase in incorporation of $\left[{ }^{3} \mathrm{H}\right]$ glucosamine into tissues and secreted glycoproteins (Table I and Fig. 2). We chose glucosamine as a precursor because it is known to be incorporated into epithelial glycoproteins in other tissues (44) and is not significantly diverted into other metabolic pathways (29). [ $\left.{ }^{3} \mathrm{H}\right]$ Glucosamine incorporation into gallbladder glycoproteins was markedly enhanced by the addition of unlabeled glucosamine, as shown by the dose-response relationship in Fig. 1. Studies in rat brain (48) indicate that glucosamine transport into cells is a saturable process with a $K_{\mathrm{m}}$ of $2.1 \mathrm{mM}$. Similarly, incorporation of $\left[{ }^{3} \mathrm{H}\right]$ glucosamine into rat intestinal slices was significantly increased by addition of unlabeled glucosamine to $18 \mathrm{mM}$ (49). In the present study, we observed a half maximal rate of incorporation of glucosamine into gallbladder glycoprotein in both control and cholesterol-fed animals at a concentration of $1 \mathrm{mM}$ glucosamine. Further, we did not observe a significant difference in the endogenous glucosamine concentration in control vs. cholesterolfed animals (Fig. 3). Taken together, these observations make it extremely unlikely that the increased gallbladder glycoprotein synthesis and secretion in the cholesterol-fed animals is attributable to a change in membrane transport or intracellular pool size of glucosamine.

The stimulatory effect of cholesterol feeding on mucin secretion appears to be specific to the gallbladder epithelium. Stomach and colon explants from 5-d cholesterol-fed animals did not manifest an increase in mucin synthesis or release in organ culture, although both of these organs would be directly exposed to the increased dietary cholesterol. Similarly, cholesterol feeding did not cause an increase in synthesis of membrane glycoproteins in gallbladder explants from cholesterol-fed animals, as indicated by the incorporation of $\left[{ }^{3} \mathrm{H}\right]$ mannose. It remains possible, however, that other cellular functions in the gallbladder mucosa not measured in our study might also 

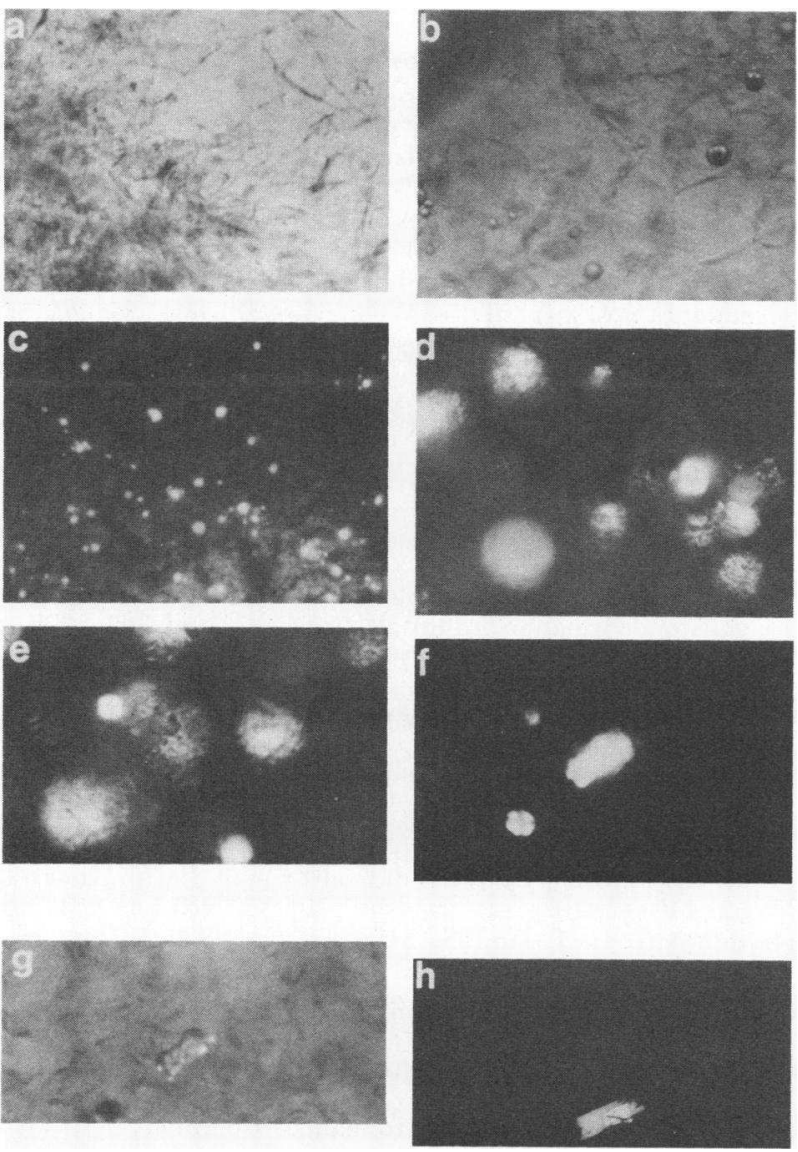

FIGURE 7 Nucleation of supersaturated hepatic bile from prairie dogs fed cholesterol for $14 \mathrm{~d}$ by human gallbladder mucin gel. (a) Mucin gel immediately after suspension in bile; (b) liquid crystals, $2 \mathrm{~h}$; (c) liquid crystals, $12 \mathrm{~h}$; (d) and (e) liquid crystals and suggestion of solid cholesterol crystals, $16 \mathrm{~h}$; (f) and (g) solid cholesterol crystals, $18 \mathrm{~h}$; (h) typical notched plate of cholesterol monohydrate (polarizing microscopy with first order quartz compensator, $\times 50$ ).

be affected by cholesterol feeding and contribute to gallstone formation.

The peak of $\left[{ }^{3} \mathrm{H}\right]$ glucosamine incorporation into gallbladder glycoproteins occurred at $5 \mathrm{~d}$, and then fell somewhat at 8 and $14 \mathrm{~d}$ (Fig. 2). The stimulus for this increase appears to exclude the influence of other known stimulants of mucus secretion such as acetylcholine (50) and cholecystokinin (51), since their effect on the gallbladder would not be inhibited by cystic duct ligation. We cannot, however, completely exclude the possibility of inadvertent damage to the blood supply or innervation of the gallbladder in these experiments. Even though mucus hypersecretion was apparent by $3 \mathrm{~d}$, the peak of mucus hypersecretion coincided with the saturation of hepatic and gallbladder bile which occurred on the 5 th $d$ of cholesterol feeding (Fig. 5), which suggests that the increased cholesterol in gallbladder bile was the stimulus for glycoprotein synthesis and secretion. The rate of incorporation of $\left[{ }^{3} \mathrm{H}\right]$ glucosamine fell appreciably at 8 and $14 \mathrm{~d}$, even though the saturation index of cholesterol had attained a constant value or increased slightly from 5 to $14 \mathrm{~d}$ (Fig. 6 ). The reason for this asynchrony between glycoprotein secretion and cholesterol saturation is unclear. One possible explanation is that some of the increased cholesterol content of bile was transferred into the apical membrane of the gallbladder epithelial cells, altering membrane properties and triggering an increased synthesis and release of mucus. For example, Neiderhiser et al. (52) have shown that micellar cholesterol is absorbed by guinea pig gallbladder epithelium. The accumulation, however, of an impermeable thick mucus gel after the 5th $d$ of cholesterol feeding may have prevented further diffusion of cholesterol into apical membranes and dampened the stimulus. Another possibility is that gallbladder mucus hypersecretion was stimulated by some constituent of bile other than cholesterol.

We were unable to demonstrate stimulation of gallbladder glycoprotein synthesis or secretion in organ culture by addition of artificial micelles containing up to $10 \mathrm{~mol} \%$ cholesterol, or native lithogenic bile reconstituted in organ culture medium. This negative result may indicate that the gallbladder epithelium must be exposed to the stimulus for $>24 \mathrm{~h}$. Unfortunately, this was not feasible in our organ culture experiments because of necrosis of epithelial cells between 36 and $48 \mathrm{~h}$ incubation. Recent studies by Axelsson et al. (53) indicate that vagal innervation may be required for gallbladder mucin secretion. Obviously, our gallbladder explants are denervated and might not respond to a secretagogue in vitro.

In previous studies of cholesterol gallstone formation in prairie dogs (23-26), dried egg yolk was the source of dietary cholesterol. It is recognized that exposure of dried cholesterol to air may result in the rapid formation of toxic autooxidative products (54). This possibility seems unlikely in the current study since we were unable to demonstrate significantly sterols other than cholesterol in hexane extracts of the lithogenic diet, gallbladder bile, or cholesterol gallstones of cholesterolfed animals. Although our gas-liquid chromatography studies suggested that cholesterol was the predominant sterol in gallbladder bile, our studies have not entirely excluded a minor oxidation product of cholesterol which might be more biologically active than cholesterol itself (53).

Our relative lipid compositions were in agreement with those reported by previous authors in prairie dogs $(23,25,26)$. In agreement with Holzbach et al. $(26,55)$, we frequently observed the presence of cholesterollecithin liquid crystals in 5- and 8-d supersaturated gallbladder biles, as we did during the in vitro nucleation studies with purified mucin gel and supersaturated hepatic bile (Fig. 7). We did not, however, specifically study the time-course of the 
transition to solid cholesterol crystals in the in vivo studies of the present work. In our high-performanceliquid-chromatography analysis of bile we did not, however, find a major qualitative or quantitative change in the major primary bile acids, taurocholate and taurochenodeoxycholate, as has been reported after more prolonged (3-6 mo) feeding (23), and which may in fact be a reflection of cholesterol ester-induced hepatotoxicity (26).

Supersaturated hepatic bile in our cholesterol-fed prairie dogs was always microscopically clear; that is, it consisted of a single micellar phase. Gallbladder bile, which on average is three times more concentrated than hepatic bile, should have an increased capacity to hold cholesterol for similar relative lipid composition (2). We nevertheless observed (Fig. 5) that gallbladder bile at 8 and $14 \mathrm{~d}$ of cholesterol feeding consisted of several phases-a clear micellar phase and a solid phase consisting of liquid crystals, solid cholesterol crystals, and stones. Furthermore, centrifuged gallbladder bile at $8 \mathrm{~d}$ was just saturated, whereas simultaneously collected hepatic bile was supersaturated. This suggests that a nucleating agent or factor in gallbladder, but not in hepatic, bile was a continuing stimulus to convert supersaturated micellar cholesterol into cholesterol precipitates. The cholesterol saturation index of homogenized gallbladder bile (containing both liquid and solid phases) was high at $8 \mathrm{~d}$ (Fig. 6), in contrast to separated bile, which fell just on the line of saturation. The difference between these two values reflects, in part, the relative amount of cholesterol nucleated in the gallbladder. The subsequent increase in the saturation of gallbladder bile at day 14 suggests that once nucleation of cholesterol occurred at $8 \mathrm{~d}$, gallbladder bile could become supersaturated.

The results of our nucleation experiments (Fig. 7) suggest that mucin gel was capable of nucleating saturated hepatic bile, in contrast to soluble protein (bovine serum albumin), which did not undergo gelation at the concentration used. In addition, we have recently demonstrated that pharmacologic inhibition of mucus secretion prevented gallstones in this model (56). Aspirin, at doses that inhibited gallbladder mucus secretion, prevented cholesterol crystals and gallstone formation in cholesterol-fed prairie dogs, despite comparable saturation indices of the gallbladder biles (56). Given the same degree of cholesterol saturation in samples of both hepatic and gallbladder biles, the formation of cholesterol liquid and solid crystals was determined by the presence of gallbladder mucus gel. On the basis of our results, we hypothesize that gallbladder mucus acts as a specific nucleating agent in the formation of cholesterol gallstones. If this hypothesis is confirmed in man, it may be possible to prevent gallstones with pharmacologic agents that inhibit mucus secretion by the gallbladder.

\section{ACKNOWLEDGMENTS}

The authors would like to thank Dr. Jerry S. Trier for his help in reviewing the histologic sections of prairie dog gallbladders; Mrs. Grace Ko, Mr. Allen Ventola, and Mr. Bradley Turner for skillful technical assistance; and Mrs. Lori Thomae, Ms. Elizabeth Steeves, and Ms. Catherine Garvin for manuscript preparation.

Grants-in-aid were generously provided by Abbott Laboratories, North Chicago, Ill., and the Cystic Fibrosis Foundation. Dr. Lee was supported by a Fogarty International Research Fellowship (5F05-TWO02663-02), Dr. LaMont by a Research Career Development Award (AM 00459) and research grant (AM 21892), and Dr. Carey by a Research Career Development Award (AM 00195) and research grant (AM 18559) all from the National Institutes of Health (U. S. Public Health Service).

\section{REFERENCES}

1. Small, D. M. 1980. Cholesterol nucleation and growth in gallstone formation (editorial). N. Engl.J. Med. 302: 13051307.

2. Carey, M. C., and D. M. Small. 1978. The physical chemistry of cholesterol solubility in bile: relationship to gallstone formation and dissolution in man. J. Clin. Invest. 61: 998-1026.

3. Holzbach, R. T., M. Marsh, M. Olszewski, and K. Holan. 1973. Cholesterol solubility in bile: evidence that supersaturated bile is frequent in healthy man. J. Clin. Invest. 52: 1467-1479.

4. Bennion, L. J., and S. M. Grundy. 1975. Effects on obesity and caloric intake on biliary lipid composition in man. J. Clin. Invest. 45: 996-1011.

5. Dowling, R. H., G. D. Bell, and J. White. 1972. Lithogentic bile in patients with ileal dysfunction. Gut. 13: 415-420.

6. Northfield, T. C., and A. F. Hofmann. 1975. Biliary lipid output during three meals and an overnight fast, I. Relationship to bile acid pool size and cholesterol saturation of bile in gallstone and control subjects. Gut. 16: 1-17.

7. Lyon, B. B. V. 1923. Non-Surgical Drainage of the Galltract. Lea \& Febiger, Philadelphia. 640 pp.

8. Shay, H., H. Tumen, and I. Rodis. 1930. The diagnosis of gallstones: relative accuracy of cholecystography and non-surgical biliary drainage. Med. Clin. N. Am. 13: 939-946.

9. Bockus, M. H., H. Shay, J. H. Willard, and J. F. Pessel. 1931. Comparison of biliary drainage and cholecystography in gallstone diagnosis with especial reference to bile microscopy. JAMA (J. Am Med. Assoc.). 96: 311-317.

10. Juniper, K., Jr., and E. N. Burson, Jr. 1957. Biliary tract studies. II. The significance of biliary crystals. Gastroenterology. 32: 175-211.

11. Holan, K. R., R. T. Holzbach, R. E. Hermann, A. M. Cooperman, and W. J. Claffey. 1979. Nucleation time: a key factor in the pathogenesis of cholesterol gallstone disease. Gastroenterology. 72: 611-617.

12. Sedaghat, A., and S. M. Grundy. 1980. Cholesterol crystals, and the formation of cholesterol gallstones. N. Engl. J. Med. 302: 1274-1277.

13. Sleisenger, M. H., and J. S. Fordtran, editors. 1979. Gastrointestinal Disease. W. B. Saunders Company, Philadelphia. 2nd edition. 1659.

14. Adler, R. D., A. L. Metzger, and S. M. Grundy. 1974. Biliary lipid secretion before and after cholecystectomy in American Indians with cholesterol gallstones. Gastroenterology. 66: 1212-1217.

15. Womack, N. A., R. Zeppa, and G. L. Irvin, III. 1963. The anatomy of gallstones. Ann. Surg. 157: 670-686. 
16. Hultén, O. 1968. Formation of gallstones. Acta Chir. Scand. 134: 557-560.

17. Womack, N. A. 1971. The development of gallstones. Surg. Gynecol. Obstet. 133: 937-945.

18. Freston, J. W., I. A. D. Bouchier, and J. Newman. 1969 Biliary mucous substances in dihydrocholesterol-induced cholelithiasis. Gastroenterology. 57: 670-678.

19. Hofmann, A. F., and E. M. Mosbach. 1964. Identification of allodeoxylcholic acid as the major component of gallstones induced in the rabbit by $5 \alpha$-cholestan3ß-ol. J. Biol. Chem. 239: 2813-2821.

20. Englert, E., C. G. Harman, J. W. Freston, R. C. Straight, and E. E. Wales. 1977. Studies on the pathogenesis of diet-induced dog gallstones. Am. J. Dig. Dis. 22: 305-312.

21. Bouchier, I. A. D., S. R. Cooperband, and B. M. El Kodsi. 1966. Mucous substances and viscosity of normal and pathological human bile. Gastroenterology. 49: 343353.

22. Lee, S. P., T. H. Lim, and A. J. Scott. 1979. Carbohydrate moieties of glycoproteins in human hepatic and gallbladder bile, gallbladder mucosa and gallstones. Clin. Sci. Mol. Med. 56: 533-538.

23. Brenneman, D. E., W. E. Conner, E. L. Forker, and L. Den Besten. 1972. The formation of abnormal bile and cholesterol gallstones from dietary cholesterol in the prairie dog. J. Clin. Invest. 51: 1495-1503.

24. Chang, S-H., K-J. Ho, and C. B. Taylor. 1973. Cholesterol gallstone formation and its regression in prairie dogs. Arch. Pathol. 96: 417-426.

25. Den Besten, L., S. Safaie-Shirazi, and W. E. Connor. 1976. Early changes in bile composition and gallstone formation induced by a high cholesterol diet in prairie dogs. Gastroenterology. 66: 1036-1045.

26. Holzbach, R. T., C. Corbusier, M. Marsh, and H. K. Naito. 1976. The process of cholesterol cholelithiasis induced by diet in the prairie dog: a physiochemical characterization. J. Lab. Clin. Med. 87: 987-998.

27. Loomis, C. R., M. J. Janiak, D. M. Small, and G. G. Shipley. 1974. The binary phase diagram of lecithin and cholesteryl linoleate. J. Mol. Biol. 86: 309-314.

28. Browning, T. H., and J. S. Trier. 1969. Organ culture of mucosal biopsies of human small intestine. J. Clin. Invest. 48: 1423-1432.

29. LaMont, J. T., and A. S. Ventola. 1977. Stimulation of colonic glycoprotein synthesis by dibutyryl cyclic AMP and theophylline. Gastroenterology. 72: 82-86.

30. Forsdyke, D. R. 1971. Application of the isotope-dilution principle to the analysis of factors affecting the incorporation of $\left({ }^{3} \mathrm{H}\right)$-glucosamine and $\left({ }^{3} \mathrm{H}\right)$-cytidine into cultured lymphocytes: evaluation of pools in serum and cultured media. Biochem. J. 125: 721-732.

31. Alpers, D. H., and G. W. Philpott. 1975. Control of deoxyribonucleic acid synthesis in normal rabbit colon mucosa. Gastroenterology. 69: 951-959.

32. Bitter, J., and H. M. Muir. 1962. A modified uronic acid carbazole reaction. Anal. Biochem. 4: 330-334.

33. Loomis, C. R., G. G. Shipley, and D. M. Small. 1979. The phase behavior of hydrated cholesterol. J. Lipid Res. 20: 525-535

34. Bartlett, G. R. 1959. Phosphorus assay in column chromatography. J. Biol. Chem. 234: 466-468.

35. Carr, J. J., and I. J. Dreckter. 1956. Simplified rapid technique for the extraction and determination of serum cholesterol without saponification. Clin. Chem. 2: 353368.

36. Abell, L. L., B. B. Levy, B. B. Brodie, and F. E. Kendal. 1952. A simplified method for the estimation of total cholesterol in serum and demonstration of its specificity. J. Biol. Chem. 195: 355-366.
37. Talalay, P. 1960. Enzymatic analysis of steroid hormones. Methods Biochem. Anal. 8: 119-143.

38. Admirand, W. H., and D. M. Small. 1968. The physicalchemical basis of cholesterol gallstone formation in man. J. Clin. Invest. 47: 1043-1052.

39. Miettinen, T. A., E. H. Ahrens, Jr., and S. M. Grundy. 1965. Quantitative isolation and gas-liquid chromatographic analysis of dietary and fecal neutral steroids. J. Lipid Res. 6: 411-424.

40. Carey, M. C. 1978. Critical tables for calculating the cholesterol saturation of native bile. J. Lipid Res. 19: 945-955.

41. Bekesi, J. G., E. Bekesi, and R. J. Winzler. 1968. Inhibitory effects of D-glucosamine and other sugars on the biosynthesis of protein, ribonucleic acid and deoxyribonucleic acid in normal and neoplastic tissues. $J$. Biol. Chem. 244: 3766-3772.

42. Sharon, N. 1975. Complex Carbohydrates. Addison-Wesley Publishing Company, Reading, Mass. 466.

43. Qureshi, R., G. C. Forstner, and J. F. Forstner. 1979. Radioimmunoassay of human intestinal goblet cell mucin. J. Clin. Invest. 64: 1149-1156.

44. LaMont, J. T., and A. S. Ventola. 1980. Synthesis and secretion of colonic glycoproteins: evidence for shedding in vivo of low molecular weight membrane components. Biochim. Biophys. Acta. 629: 553-565.

45. Van der Linden, W. 1979. Gallstone formation in animals. In Gallstones. M. M. Fisher, C. A. Goresky, E. A Shaffer, and S. M. Strasberg, editors. Plenum Publishing Corp., New York. 213-222.

46. Peng, S. K., P. Tham, C. B. Taylor, and B. Mikkelson. 1979. Cytotoxicity of oxidation derivatives of cholesterol on cultured aortic smooth muscle cells and their effect on cholesterol biosynthesis. Am. J. Clin. Nutr. 32: 10331042.

47. Chicoye, E., W. D. Powrie, and O. Fennema. 1968. Photo-oxidation of cholesterol in spray-dried egg yolk upon irradiation. J. Food Sci. 33: 581-588.

48. Tan, C. H., N. A. Peterson, and E. Raghupathy. 1977. D-Glucosamine uptake by rat brain synaptosomes. Biochim. Biophys. Acta. 464: 459-462.

49. Lukie, B. E., and G. G. Forstner. 1972. Synthesis of intestinal glycoprotein: incorporation of $\left(1-{ }^{14} \mathrm{C}\right) \mathrm{glu}$ cosamine in vitro. Biochim. Biophys. Acta. 261: 353-364.

50. Chakrin, L. N., A. P. Baker, P. Christian, and J. R Wardell. 1973. Effect of cholinergic stimulation on the release of macromolecules by canine trachea in vitro. Am. Rev. Respir. Dis. 108: 69-76.

51. Wahlin, T., G. D. Bloom, and A. Danielsson. 1976. Effect of cholecystokinin pancreozymin (CCK-PZ) on glycoprotein secretion from mouse gallbladder epithelium. Cell Tissue Res. 171: 425-435.

52. Neiderhiser, D. H., C. K. Harmon, and H. P. Roth. 1976. Absorption of cholesterol by the gallbladder. J. Lipid Res. 17: 117-124.

53. Axelsson, H., A. Danielsson, R. Henriksson, and T. Wahlin, 1979. Secretory behavior and ultrastructural changes in mouse gallbladder principal cells after stimulation with cholinergic and adrenergic drugs. Gastroenterology. 76: 335-340.

54. Editorial. 1980. Atherosclerosis and auto-oxidation of cholesterol. Lancet. I: 964-965.

55. Holzbach, R. T., and C. Corbusier. 1978. Liquid crystals and cholesterol nucleation during equilibration in supersaturated bile analogs. Biochim. Biophys. Acta. 528: 436444.

56. Lee, S. P., J. T. LaMont, and M. C. Carey. 1981. Aspirin prevention of cholesterol gallstone formation in prairie dogs. Science (Wash. D. C.). 211: 1429-1432. 